\title{
The Low Energy Scattering for Slowly Decreasing Potentials
}

\author{
D. R. Yafaev
}

Leningrad Department of Mathematical Institute, 27, r. Fontanka, 191011, Leningrad, USSR

\begin{abstract}
For the radial Schrödinger equation with a potential $q(x)$ decreasing at infinity as $q_{0} x^{-\alpha}, \alpha \in(0,2)$, the low energy asymptotics of spectral and scattering data is found. In particular, it is shown that for $q_{0}>0$ the spectral function vanishes exponentially as the energy $k^{2}$ tends to zero. On the contrary, there is always a zero-energy resonance for $q_{0}<0$. These results determine the local asymptotics of solutions of the time-dependent Schrödinger equation for large times $t$. Specifically, for positive potentials its solutions decay as $\exp \left(-\vartheta_{0} t^{(2-\alpha) /(2+\alpha)}, \vartheta_{0}>0, t \rightarrow \infty\right.$. In the case $\alpha \in(1,2)$ it is shown that for $\pm q_{0}>0$ the phase shift tends to $\pm \infty$ as $k \rightarrow 0$ and its asymptotics is evaluated.
\end{abstract}

\section{Introduction}

In the study of the low energy scattering of nonrelativistic particles it is usually assumed that the potential is vanishing sufficiently quickly at infinity. Moreover, in the physics literature potentials are often assumed to be central. In this case the low energy scattering is determined $[1,2]$ only by the behaviour of particles with a zero angular momentum $\ell$. For $\ell=0$ the phase shift and generically the partial cross section have finite limits as the energy tends to zero. This shows that scattering at low energies depends weakly on the shape of the potential. Specifically, low energy scattering is well described in terms of the scattering length. Recently the low energy asymptotics of scattering data was found and rigorously proved by A. Jensen and T. Kato [3] for arbitrary quickly decreasing potentials. The behaviour at low energies of a spectral family of the corresponding Hamiltonian was also investigated in [3]. Hence the local decay in time of the solution of the time-dependent Schrödinger equation is deduced. Namely, the solution decays $[4,5,3]$ generically as $t^{-3 / 2}$ if the initial state is sufficiently localized in the space and is orthogonal to the bound states. Most of these results are valid if $q(\mathbf{x})=O\left(|\mathbf{x}|^{-2-\varepsilon}\right), \varepsilon>0$. We call such potentials quickly decreasing or short-range.

In this paper it is found that for potentials vanishing slower than $|\mathbf{x}|^{-2}$ as $|\mathbf{x}|$ $\rightarrow \infty$, the low energy behaviour of spectral and scattering data is essentially different 
from a short-range case. We restrict ourselves to scattering by a central potential $q(x), x=|\mathbf{x}|$, for fixed $\ell$. Some qualitative results, valid for arbitrary (not only central) slowly decreasing potentials in any dimension of the space, were published in [6]. For simplicity we take $\ell=0$, though our results may be literally carried over to all $\ell$. We denote by $H$ the Hamiltonian corresponding to a differential operator $-d^{2} / d x^{2}+q(x)$ and a boundary condition $u(0)=0$.

If $q(x)=O\left(x^{-1-\varepsilon}\right), \varepsilon>0$, then the radial Schrödinger equation

$$
-u^{\prime \prime}+q(x) u=s^{2} u
$$

has for $\operatorname{Im} s \geqq 0, s \neq 0$, the Jost solution $f(x, s)$ defined by its asymptotics as $x \rightarrow \infty$ :

$$
f(x, s) \sim e^{i s x} .
$$

It is well known that in a radial case all characteristics of scattering are expressed in terms of the Jost function $M(s)=f(0, s)$ for $s>0$. We shall write $k$ instead of $s$ if $s$ is positive; $k^{2}$ is the energy of the quantum particle. Specifically, the argument of $M(k)$ is the phase shift $\eta(k)$ and $A(k)=|M(k)|$ determines the spectral function

$$
\rho(k)=2 \pi^{-1} A(k)^{-2} k^{2}
$$

of the Hamiltonian $H$, which is interpreted as the density of quantum states of the continuous spectrum at the energy $k^{2}$. For potentials vanishing at infinity slower than Coulomb, i.e. for $q(x) \sim q_{0} x^{-\alpha}, \alpha \in(0,1], x \rightarrow \infty$, the canonical definition of the Jost function $M(k)$ is lacking but its modulus $A(k)$ does not depend on the choice of regularization. We recall that in a short-range case when $q(x)=O\left(x^{-2-\varepsilon}\right), \varepsilon>0$, the function $M(s)$ is continuous as $s \rightarrow 0$ and generically $M(0) \neq 0$. The equality $M(0)=0$ is interpreted as an appearance of a zero-energy resonance for the Hamiltonian $H$. In the presence of such a resonance the low energy behaviour of scattering data is slightly different from a generic case. The notion of zero-energy resonance is discussed in $[3,7]$.

The aim of the present paper consists in deriving the asymptotics of $M(k)$ as $k \rightarrow 0$ for slowly decreasing potentials when $q(x) \sim q_{0} x^{-\alpha}, \alpha \in(0,2)$, at infinity. It appears that for such potentials the phase shift is unbounded as $k \rightarrow 0$ :

$$
\eta(k) \sim \eta_{0} k^{1-2 / \alpha}, \alpha \in(1,2),
$$

where $\eta_{0}<0$ for $q_{0}<0$ and $\eta_{0}>0$ for $q_{0}>0$. The behaviour of $A(k)$ as $k \rightarrow 0$ depends essentially on the sign of the potential:

$$
\left.\begin{array}{l}
A(k) \sim A_{-} k^{1 / 2}, q_{0}<0, \\
A(k) \sim A_{+} k^{1 / 2} \exp \left(g_{0} k^{1-2 / \alpha}\right), g_{0}>0, q_{0}>0 .
\end{array}\right\}
$$

The formula (1.4) shows that the scattering matrix and the partial scattering cross section are oscillatory as $k \rightarrow 0$. We emphasize that (1.4) permits us to recover information about the behaviour of $q(x)$ at infinity. The first formula (1.5) ensures that $M(0)=0$, i.e. there is always a zero-energy resonance in case $q_{0}<0$. The second formula (1.5) provides an exponential fall-off of the spectral function as $k \rightarrow 0$. This result may be interpreted as a virtual shift of the continuous spectrum; the spectral point zero turns out to be in a sense quasiregular in this case. The exponential fall-off as $k \rightarrow 0$ of the density of the states of the continuous spectrum in case $q_{0}>0$ should 
be compared with an appearance of an infinite number of the discrete states in the case $q_{0}<0$. The asymptotic formula for $\rho(k)$ (see (5.11)) plays a role of the classical Weyl's formula for the function of distribution of discrete eigenvalues.

Formulae (1.5) enable one to find the asymptotics of solutions of the timedependent Schrödinger equation

$$
i \frac{\partial u}{\partial t}=H u, \quad u(x, 0)=u_{0}(x)
$$

for fixed $x$ and $t \rightarrow \infty$. Let the initial state $u_{0}$ be orthogonal to the bound states and have compact support. Then the wave function $u(x, t)$ vanishes locally as $t^{-1}, t \rightarrow \infty$ for negative potentials, and as $\exp \left(-\vartheta_{0} t^{(2-\alpha) /(2+\alpha)}\right), \vartheta_{0}>0$ for positive potentials. The precise proof of the last assertion requires an analyticity of the spectral function $\rho(s)$ and the study of its behaviour as $s \rightarrow 0$ in some sector of a complex plane. This study may be achieved for analytic (for sufficiently large $x$ ) potentials $q(x)$.

The proof of the asymptotic formula for the Jost function is based on an investigation of a quasiclassical solution $\psi(x, s)$ of Eq. (1.1). We construct $\psi(x, s)$ with the help of the Green-Liouville approximation, which in the physics literature is usually called the WKB-method (see e.g. [8]). It turns out that for slowly decreasing potentials the quasiclassical solution $\psi(x, s)$ (in contrast to $f(x, s)$ ) is continuous as $s \rightarrow 0$ ( $x$ fixed). Thus for such potentials the small energy is the correct parameter of the quasiclassical approximation. The explicit expressions for the constants $\eta_{0}$ and $g_{0}$ (see Sect. 7) confirm that formulae (1.4), (1.5) are essentially of a quasiclassical nature.

This paper is organized as follows. In Sect. 2 for potentials vanishing at infinity in a sufficiently arbitrary way, the solution $\psi(x, s)$ is introduced and investigated outside some neighbourhood of the point $s=0$. Here the spectral function $\rho(k)$ is expressed in terms of $\psi(0, k)$ and an appropriate theorem on eigen-function expansion for the operator $H$ is obtained. The behaviour of $\psi(x, s)$ as $s \rightarrow 0$ is studied in Sect. 3. In Sect. 4 the analytic continuation of $\rho(s)$ in some sector of a complex plane is constructed and in Sect. 5 the asymptotics of $\rho(s)$ as $s \rightarrow 0$ is evaluated. These results are used in Sect. 6 to find the local asymptotics of solutions of the timedependent Schrödinger equation. In Sect. 7 we study potentials satisfying $q(x)$ $=O\left(x^{-1-\varepsilon}\right), \varepsilon>0$, when scattering data have unequivocal meaning. Here the connection between solutions $f(x, s)$ and $\psi(x, s)$ is established and the asymptotics of the function $M(s)$ as $s \rightarrow 0$ is derived. The general discussion of our results is also contained in Sect. 7.

\section{Quasiclassical Solutions of the Schrödinger Equation}

Let us consider Eq. (1.1) for $x \geqq 0$ and $\operatorname{Im} s \geqq 0, s \neq 0$. It is everywhere assumed that $q(x)=\overline{q(x)}, q(x) \rightarrow 0$ as $x \rightarrow \infty$ and $x q(x) \in L_{1}\left(0, x_{1}\right)$ for all $x_{1}<\infty$. This condition admits singularities of $q(x)$ of the type $c x^{-\beta}, \beta<2$, as $x \rightarrow 0$.

At first we shall recall some well-known facts about regular solutions $\varphi(x, s), \theta(x, s), s \in \mathbb{C}$, of $(1.1)$, which are defined by

$$
\varphi\left(a_{0}, s\right)=0, \varphi^{\prime}\left(a_{0}, s\right)=1, \theta\left(a_{0}, s\right)=1, \theta^{\prime}\left(a_{0}, s\right)=0
$$


for some fixed number $a_{0}>0$. The dependence of $\varphi$ and $\theta$ on $a_{0}$ is dropped out of notation. The number $a_{0}$ is chosen positive because of a possible singularity of $q(x)$ at $x=0$. Equation (1.1) also has a unique solution $\varphi_{0}(x, s)$ satisfying $\varphi_{0}(0, s)=0$, $\varphi_{0}^{\prime}(0, s)=1$. It is well known that $\varphi(x, s), \varphi^{\prime}(x, s), \theta(x, s), \theta^{\prime}(x, s), \varphi_{0}(x, s), \varphi_{0}^{\prime}(x, s)$ for every fixed $x>0$ are entire functions of a variable $s$. For all $s \in \mathbb{C}$ functions $\varphi(x, s), \theta(x, s)$ have limits as $x \rightarrow 0$ and $\varphi(0, s), \theta(0, s)$ are also entire functions of $s$. For derivatives relations

$$
\lim _{x \rightarrow 0} x \varphi^{\prime}(x, s)=0, \lim _{x \rightarrow 0} x \theta^{\prime}(x, s)=0
$$

hold. For every $x \geqq 0$ the following asymptotics as $|s| \rightarrow \infty$ are valid:

$$
\varphi(x, s) \sim s^{-1} \sin s\left(x-a_{0}\right), \theta(x, s) \sim \cos s\left(x-a_{0}\right), \varphi_{0}(x, s) \sim s^{-1} \sin s x .
$$

Formulae (2.2) may be differentiated with respect to $x$ for $x>0$.

We need notation for sectors in a complex plane of $s$. Let

$$
\begin{aligned}
Y\left(\omega_{1}, \omega_{2}\right) & =\left\{s \in \mathbb{C}: \omega_{1}<\arg s<\omega_{2}\right\}, Y\left[\omega_{1}, \omega_{2}\right]=\left\{s \in \mathbb{C}: \omega_{1} \leqq \arg s \leqq \omega_{2}\right\}, \\
Y_{\varepsilon}\left(\omega_{1}, \omega_{2}\right) & =Y\left(\omega_{1}, \omega_{2}\right) \cap\{|s|<\varepsilon\}, Y^{\varepsilon}\left(\omega_{1}, \omega_{2}\right)=Y\left(\omega_{1}, \omega_{2}\right) / Y_{\varepsilon}\left(\omega_{1}, \omega_{2}\right) .
\end{aligned}
$$

We everywhere suppose that the branch of any function $z^{\gamma}$ is fixed by the condition $\arg z \in[-\pi, \pi] ; C$ and $c$ are generic constants. To construct a quasiclassical solution $\psi(x, s)$, we assume that for some $x_{0} \geqq 0$ and $x>x_{0}$ the potential $q(x)$ is twice differentiable and

$$
\int_{x_{0}}^{\infty}\left(\left|q^{\prime \prime}(x)\right|+\left|q^{\prime}(x)\right|^{2}\right) d x<\infty .
$$

This implies that $q^{\prime}(x) \rightarrow 0$ as $x \rightarrow 0$. The condition (2.3) admits sufficiently arbitrary decay of $q(x)$ as $x \rightarrow \infty$. Let us introduce some auxiliary functions: $Q(x, s)=q(x)-s^{2}$,

$$
\begin{aligned}
\zeta(x, s) & =(32)^{-1}\left[4 q^{\prime \prime}(x) Q(x, s)^{-3 / 2}-5 q^{\prime}(x)^{2} Q(x, s)^{-5 / 2}\right], \\
\xi(a, x ; s) & =\int_{a}^{x} Q(y, s)^{1 / 2} d y .
\end{aligned}
$$

Then $\operatorname{Re} Q(x, s)^{1 / 2} \geqq 0$ and for $x \geqq x_{0}$ the function $Q(x, s)^{1 / 2}$ is analytic in $s \in Y(0, \pi / 2) \cup Y(\pi / 2, \pi)$ and is continuous in corresponding closed sectors. Note that $Q(x, s)^{1 / 2}$ is not in general continuous as $s$ goes over the positive part of the imaginary axis.

The solution $\psi(x, s)$ of Eq. (1.1), where $s \in Y[0, \pi / 2] \cup Y[\pi / 2, \pi], s \neq 0$, is called quasiclassical if

$$
\left.\begin{array}{rl}
\psi(x, s) & \sim Q^{-1 / 4}(x, s) \exp (-\xi(a, x ; s)), \\
\psi^{\prime}(x, s) & \sim-Q^{1 / 4}(x, s) \exp (-\xi(a, x ; s))
\end{array}\right\}
$$

as $x \rightarrow \infty$. We fix $a \geqq x_{0}$ and omit the dependence of $\psi$ on $a$. Formulae (2.4) may of course be simplified

$$
\left.\begin{array}{rl}
\psi(x, s) & \sim(-i s)^{-1 / 2} \exp (-\xi(a, x ; s)) \\
\psi^{\prime}(x, s) & \sim-(-i s)^{1 / 2} \exp (-\xi(a, x ; s))
\end{array}\right\} .
$$


However, for slowly decreasing potentials (2.4) is uniform in $s, s \rightarrow 0$, whereas (2.5) does not have this property.

Our construction of $\psi(x, s)$ relies on the study of an integral equation for functions $u_{j}(x, s), j=1,2$, connected with $\psi(x, s)$ by

$$
\left(\frac{\psi}{\psi^{\prime}}\right)=Q^{-1 / 4}(x, s) e^{-\xi(a, x ; s)}\left(\begin{array}{cc}
1 & 1 \\
Q^{1 / 2}-q^{\prime}(4 Q)^{-1} & -Q^{1 / 2}-q^{\prime}(4 Q)^{-1}
\end{array}\right)\left(\begin{array}{l}
u_{1} \\
u_{2}
\end{array}\right) .
$$

Namely, let us consider the following system

$$
\left.\begin{array}{l}
u_{1}(x, s)=-\int_{x}^{\infty} e^{-2 \xi(x, y ; s)} \zeta(y, s)\left[u_{1}(y, s)+u_{2}(y, s)\right] d y, \\
u_{2}(x, s)=1+\int_{x}^{\infty} \zeta(y, s)\left[u_{1}(y, s)+u_{2}(y, s)\right] d y
\end{array}\right\} .
$$

For sufficiently large $x$ the system (2.7) may be solved by iterations, i.e.

$$
u_{j}(x, s)=\sum_{n=0}^{\infty} u_{j}^{(n)}(x, s),
$$

where $u_{1}^{(0)}=0, u_{2}^{(0)}=1$, and for $n \geqq 1$

$$
\left.\begin{array}{l}
u_{1}^{(n)}(x, s)=-\int_{x}^{\infty} \exp (-2 \xi(x, y ; s)) \zeta(y, s)\left[u_{1}^{(n-1)}(y, s)+u_{2}^{(n-1)}(y, s)\right] d y, \\
u_{2}^{(n)}(x, s)=\int_{x}^{\infty} \zeta(y, s)\left[u_{1}^{(n-1)}(y, s)+u_{2}^{(n-1)}(y, s)\right] d y
\end{array}\right\}
$$

Theorem 1. Let the condition (2.3) hold and $\operatorname{Im} s \geqq 0,|s| \geqq \varepsilon>0$. Then for sufficiently large $x_{1}=x_{1}(\varepsilon)$ and $x \geqq x_{1}$ the system (2.7) has a unique solution $u_{j}(x, s), j=1,2$, which is bounded on $\left(x_{1}, \infty\right)$. This solution is obtained by formulae (2.8), (2.9). For each $x \geqq x_{1}$ functions $u_{j}(x, s), j=1,2$, are analytic in $s$ for $\operatorname{Im} s>0,|s|>\varepsilon$ and are continuous in $s$ for $\operatorname{Im} s \geqq 0,|s| \geqq \varepsilon$. Moreover,

$$
\lim _{x \rightarrow \infty} u_{1}(x, s)=0, \quad \lim _{x \rightarrow \infty} u_{2}(x, s)=1
$$

uniformly in $s,|s| \geqq \varepsilon$, and for each $x \geqq x_{1}$

$$
\lim _{|s| \rightarrow \infty} u_{1}(x, s)=0, \quad \lim _{|s| \rightarrow \infty} u_{2}(x, s)=1 .
$$

Proof. Since $q(x) \rightarrow 0$ as $x \rightarrow \infty$,

$$
|Q(x, s)| \geqq c(\varepsilon)>0
$$

for $|s| \geqq \varepsilon$ and $x \geqq x_{1}=x_{1}(\varepsilon)$. It follows that

$$
|\zeta(x, s)| \leqq C(\varepsilon)\left(\left|q^{\prime \prime}(x)\right|+\left|q^{\prime}(x)\right|^{2}\right) .
$$

Once $\operatorname{Re} \xi(x, y ; s) \geqq 0$

$$
|\exp (-2 \xi(x, y ; s))| \leqq 1
$$


Let us solve the system (2.7) by iterations. According to (2.13) and (2.14), formulae (2.9) ensure a recurrent estimate

$$
\left|u_{j}^{(n)}(x, s)\right| \leqq C(\varepsilon) \int_{x}^{\infty}\left(\left.q^{\prime \prime}(y)|+| q^{\prime}(y)\right|^{2}\right)\left(\left|u_{1}^{(n-1)}(y, s)\right|+\left|u_{2}^{(n-1)}(y, s)\right|\right) d y,
$$

whence

$$
\left|u_{j}^{(n)}(x, s)\right| \leqq C^{n}(\varepsilon)(n !)^{-1}\left[\int_{x}^{\infty}\left(\left|q^{\prime \prime}(y)\right|+\left|q^{\prime}(y)\right|^{2}\right) d y\right]^{n} .
$$

Thus by condition (2.3) the series (2.9) is convergent for $x \geqq x_{1}$ and therefore it determines the unique bounded solution of the system (2.7). The estimate (2.15) ensures relations (2.10). Since a constant $C(\varepsilon)$ in (2.13), (2.15) vanishes as $\varepsilon \rightarrow \infty,(2.11)$ also holds. The analyticity and the continuity of $u_{j}(x, s)$ follow from corresponding properties of functions $\zeta(x, s), \xi(x, y ; s)$ and (2.12)-(2.14).

Defining $\psi(x, s)$ by $(2.6)$ it is easy now to prove the following

Theorem 2. Let the condition (2.3) hold and $s \in Y[0, \pi / 2] \cup Y[\pi / 2, \pi], s \neq 0$. Then Eq.(1.1) has a solution $\psi(x, s)$ obeying (2.4) as $x \rightarrow \infty$. For each $\varepsilon>0$ the asymptotics (2.4) are uniform in $s \in Y^{\varepsilon}[0, \pi / 2] \cup Y^{\varepsilon}[\pi / 2, \pi]$. For fixed $x \geqq 0$ and $|s| \rightarrow \infty, \operatorname{Im} s \geqq 0$,

$$
\psi(x, s) \sim(-i s)^{-1 / 2} \exp [i s(x-a)] .
$$

The relation (2.16) may be differentiated with respect to $x, x>0$. The functions $\psi(x, s)$ for $x \geqq 0$ and $\psi^{\prime}(x, s)$ for $x>0$ are analytic in $s \in Y(0, \pi / 2) \cup Y(\pi / 2, \pi)$ and are continuous in $s \in Y[0, \pi / 2] \cup Y[\pi / 2, \pi]$, with an exception of point $s=0$.

Proof. Let $|s| \geqq \varepsilon$, Im $s \geqq 0$ and $u_{j}, j=1,2$, be the solution of (2.7), constructed in Theorem 1. By differentiation it is verified that functions $u_{j}$ satisfy for $x \geqq x_{1}(\varepsilon)$ the system

$$
\left(\begin{array}{l}
u_{1} \\
u_{2}
\end{array}\right)^{\prime}=\left(\begin{array}{cc}
2 Q^{1 / 2}+\zeta & \zeta \\
-\zeta & -\zeta
\end{array}\right)\left(\begin{array}{l}
u_{1} \\
u_{2}
\end{array}\right) .
$$

Substitution (2.6) ensures that in terms of $\psi, \psi^{\prime}$ this system takes the form

$$
\left(\begin{array}{c}
\psi \\
\psi^{\prime}
\end{array}\right)^{\prime}=\left(\begin{array}{ll}
0 & 1 \\
Q & 0
\end{array}\right)\left(\begin{array}{c}
\psi \\
\psi^{\prime}
\end{array}\right)
$$

which is obviously equivalent to (1.1). All properties of $\psi(x, s)$ and $\psi^{\prime}(x, s)$ for $|s| \geqq \varepsilon$ and $x \geqq x_{1}(\varepsilon)$ are immediate consequences of Theorem 1. For the proof of these properties for all $x$ we use additionally the representation

$$
\psi(x, s)=b(s) \varphi(x, s)+d(s) \theta(x, s)
$$

where $b(s)=w(\psi(., s), \theta(., s)) \equiv \psi^{\prime}(x, s) \theta(x, s)-\psi(x, s) \theta^{\prime}(x, s), d(s)=-w(\psi(., s), \varphi(., s))$. Evaluating these Wronskions for $x \geqq x_{1}(\varepsilon)$, we find that $b(s)$ and $d(s)$ are analytic in $Y^{\varepsilon}(0, \pi / 2) \cup Y^{\varepsilon}(\pi / 2, \pi)$ and are continuous in the closure of this domain. By (2.17) $\psi(x, s), x \geqq 0$, and $\psi^{\prime}(x, s), x>0$, have the same properties in a variable $s$, since $\varphi(x, s)$ and $\theta(x, s)$ are entire functions of $s$. Formula (2.16) and the corresponding relation for $\psi^{\prime}(x, s)$ follow quite similarly from their validity for $x \geqq x_{1}(\varepsilon)$ and (2.2). Since $\varepsilon$ is arbitrary, this concludes the proof. 
Remark. The functions $\psi(x, s), \psi^{\prime}(x, s)$ are not continuous as $s$ goes over the ray arg $s$ $=\pi / 2$, because $\xi(a, x ; s), x \geqq a$, has in general different limits from the right and from the left. Still if $|s|$ is sufficiently large, namely $|s|^{2}>-\inf _{x \geq a} q(x)$, then $\xi(a, x ; s)$ and therefore $\psi(x, s), \psi^{\prime}(x, s)$ are continuous in the neighbourhood of the ray arg $s=\pi / 2$. Specifically, for potentials positive on $\left(x_{0}, \infty\right)$, the functions $\psi(x, s, x) \geqq 0$, and $\psi^{\prime}(x, s), x>0$, are analytic in the whole upper half-plane $\operatorname{Im} s>0$.

Under the assumptions of this section the operator (the Hamiltonian of the quantum particle) $H=-d^{2} / d x^{2}+q(x)$ with the boundary condition $u(0)=0$ is selfadjoint in the Hilbert space $L_{2}(0, \infty)$. Since $q(x) \rightarrow 0$ as $x \rightarrow \infty$, the continuous spectrum of $H$ coincides with the positive half-line and its negative spectrum consists of eigenvalues, which may accumulate only at the point zero. Let $R_{z}=(H-z)^{-1}$, Im $z \neq 0$, be a resolvent of the operator $H$ and $E_{\lambda}, \lambda \in \mathbb{R}$, be its spectral family.

Now we shall establish an eigenfunction expansion for the Hamiltonian $H$ in terms of the quasiclassical solutions $\psi(x, s)$. Since $\psi(., s) \in L_{2}(0, \infty)$ for $\operatorname{Im} s>0$, the kernel $R_{z}(x, y)$ of $R_{z}$ obeys

$$
R_{z}(x, y)=w\left(\varphi_{0}(., s), \psi(., s)\right)^{-1} \varphi_{0}(x, s) \psi(y, s), x \leqq y, z=s^{2}, \operatorname{Im} s>0,
$$

and $R_{z}(x, y)=R_{z}(y, x)$. Conditions $(2.1), \varphi_{0}(0, s)=0, \varphi_{0}^{\prime}(0, s)=1$ imply that

$$
w\left(\varphi_{0}(., s), \psi(., s)\right)=\psi(0, s) .
$$

Note that the function (2.18) is meromorphic in $s$ for $\operatorname{Im} s>0$, because $\psi(0, s)^{-1} \psi(y, s)$ is continuous at the ray $\arg s=\pi / 2$. The equality $\psi(0, s)=0$ for $\operatorname{Im} s$ $>0$ ensures that $z=s^{2}$ is an eigenvalue of $H$. Thus complex zeros of $\psi(0, s)$ lie on the imaginary axis. The asymptotics $(2.5)$ show that $\psi(0,-k)=\overline{\psi(0, k)}$ for $k>0$ and

$$
\left.\begin{array}{l}
v(k) \equiv(2 i)^{-1} w(\psi(., k), \psi(.,-k))=\exp (-2 g(k)), \\
g(k)=\int_{a}^{\infty} Q(y, k)_{+}^{1 / 2} d y,
\end{array}\right\}
$$

where $Q_{+}=\max \{Q, 0\}$. Specifically, the functions $\psi(x, k)$ and $\psi(x,-k)$ are linearly independent. The equalities (2.19) and $\varphi_{0}(x,-s)=\varphi_{0}(x, s)$ imply that

$$
\varphi_{0}(x, k)=(2 i v(k))^{-1}[\psi(0,-k) \psi(x, k)-\psi(0, k) \psi(x,-k)] .
$$

By $(2.21) \psi(0, k) \neq 0$, and hence the function $R_{z}(x, y)$ is continuous in $z$ in the neighbourhood of the cut over $[0, \infty)$ with the exception of the point $z=0$. Thus the positive spectrum of $H$ is absolutely continuous. The formula $2 \pi i d E_{\lambda} / d \lambda=R_{\lambda+i 0}$ $-R_{\lambda-i 0}$ permits us to evaluate $d E_{\lambda} / d \lambda$. Namely, taking into account (2.18), (2.19) and (2.21), we find that

$$
\frac{d E_{\lambda}(x, y)}{d \lambda}=(2 k)^{-1} \rho(k) \varphi_{0}(x, k) \varphi_{0}(y, k), \quad \lambda=k^{2},
$$

where

$$
\rho(k)=2 \pi^{-1} k v(k)[\psi(0, k) \psi(0,-k)]^{-1}
$$

is called the spectral function of the operator $H$. The function $\rho$ does not evidently depend on $a$. Thus we have proved the following 
Theorem 3. Let the condition (2.3) be fulfilled and let the function $\rho$ be defined by (2.20), (2.23). Then the derivative of the spectral family of $H$ obeys (2.22). Moreover, $\rho(k)$ is continuous for $k>0$ and $\rho(k) \sim 2 \pi^{-1} k^{2}, k \rightarrow \infty$.

Note that an eigenfunction expansion for the operator $H$ with a long-range potential was established earlier in [9]. However, in [9] the spectral function was expressed in terms of modified Jost solutions rather than quasiclassical ones. This does not permit us to use directly the results of [9].

\section{Slowly Decreasing Potentials. The Continuity of Quasiclassical Solutions as $\boldsymbol{s} \rightarrow \mathbf{0}$}

As was already noted in the introduction, potentials vanishing at infinity slower than $x^{-2}$ are called slowly decreasing in the present paper. More precisely, we assume in this section that $q(x) \neq 0$ for $x \geqq x_{0}\left(x_{0}\right.$ is as before some nonnegative number) and

$$
\left.\begin{array}{r}
\int_{x_{0}}^{\infty}\left[\left|q^{\prime \prime}(x)\right||q(x)|^{-3 / 2}+\left|q^{\prime}(x)\right|^{2}|q(x)|^{-5 / 2}\right] d x<\infty \\
\lim _{x \rightarrow \infty} q^{\prime}(x)|q(x)|^{-3 / 2}=0 .
\end{array}\right\}
$$

The condition (3.1) corresponds to potentials vanishing as $q_{0} x^{-\alpha}, \alpha \in(0,2)$, at infinity.

Let us extend the definition (2.4) of a quasiclassical solution to a point $s=0$. Namely, we introduce the solution $\psi_{0}(x)$ of $(1.1)$, where $s=0$, with the asymptotics

$$
\begin{aligned}
& \psi_{0}(x) \sim q(x)^{-1 / 4} \exp \left[-\int_{a}^{x} q(y)^{1 / 2} d y\right], \\
& \psi_{0}^{\prime}(x) \sim-q(x)^{1 / 4} \exp \left[-\int_{a}^{x} q(y)^{1 / 2} d y\right], \quad x \rightarrow \infty .
\end{aligned}
$$

Here it is supposed that $\arg q=0$ if $q>0$ and $\arg q=-\pi$ if $q<0$. Then

$$
\left.\begin{array}{l}
q(x)^{\gamma}=\lim _{s \rightarrow 0} Q(x, s)^{\gamma}(q(x)>0, \operatorname{Im} s \geqq 0 \text { or } q(x)<0, s \in Y[0, \pi / 2]), \\
\overline{q(x)^{\gamma}}=\lim _{s \rightarrow 0} Q(x, s)^{\gamma}(q(x)<0, s \in Y[\pi / 2, \pi])
\end{array}\right\} .
$$

Theorem 4. Let the condition (3.1) hold. Then the equation $-\psi_{0}^{\prime \prime}+q(x) \psi_{0}=0$ has the solution obeying (3.2). If $q(x)>0, x \geqq x_{0}$, then the asymptotics (2.4) is uniform in $s \in Y[\delta, \pi-\delta]$ (the point $s=0$ included), where $\delta$ is an arbitrary positive number. For each $x \geqq 0$

$$
\lim _{s \rightarrow 0} \psi(x, s)=\psi_{0}(x), \quad s \in Y[\delta, \pi-\delta] .
$$

If $q(x)<0, x \geqq x_{0}$, then (2.4) is uniform in $\mathrm{s} \in \mathrm{Y}[0, \pi / 2-\delta] \cup Y[\pi / 2+\delta, \pi]$. For each $x \geqq 0$

$$
\lim _{s \rightarrow 0} \psi(x, s)=\psi_{0}(x), s \in Y[0, \pi / 2-\delta], \lim _{s \rightarrow 0} \psi(x, s)=\overline{\psi_{0}(x)}, s \in Y[\pi / 2+\delta, \pi] .
$$


Relations (3.4), (3.5) may be differentiated in $x$ for $x>0$.

Proof. Recall that for sufficiently large $x, x>x_{1}(|s|)$, the functions $\psi(x, s)$ and $\psi^{\prime}(x, s)$ were defined by (2.6), where $u_{j}$ satisfy (2.7). Note that for $x \geqq x_{0}$ in cases $q(x)$ $>0, s \in Y[\delta, \pi-\delta]$ or $q(x)<0, s \in Y[0, \pi / 2-\delta] \cup Y[\pi / 2+\delta, \pi]$ the estimate

$$
|Q(x, s)| \geqq c|q(x)| \text {. }
$$

holds. The constant $c$ here depends only on $\delta$. Thus the function $\zeta(x, s)$ obeys

$$
|\zeta(x, s)| \leqq C\left(\left|q^{\prime \prime}(x)\right||q(x)|^{-3 / 2}+\left|q^{\prime}(x)\right|^{2}|q(x)|^{-5 / 2}\right) .
$$

The following copies the proofs of Theorems 1 and 2. Namely, by (3.7) and (2.14) for $x \geqq x_{0}, j=1,2$

$$
\left|u_{j}^{(n)}(x, s)\right| \leqq C^{n}(n !)^{-1}\left[\int_{x}^{\infty}\left(\left|q^{\prime \prime}(y)\right||q(y)|^{-3 / 2}+\left|q^{\prime}(y)\right|^{2}|q(y)|^{-5 / 2}\right) d y\right]^{n},
$$

where $C$ does not depend on $|s|$. Thus the series (2.8) converges for $x \geqq x_{0}$ uniformly in $s \in Y[\delta, \pi-\delta]$ (if $q(x)>0$ ) and in $s \in Y[0, \pi / 2-\delta] \cup Y[\pi / 2+\delta, \pi]$ (if $q(x)<0$ ). Moreover, formulae (2.10) and hence (2.4) are also uniform in $s$. In particular, for $s=0$ this proves the existence of $\psi_{0}(x)$. For $x \geqq x_{0}$ equalities (3.4), (3.5) and corresponding relations for $\psi^{\prime}(x, s)$ follow directly from (3.3). With the help of (2.17) this can be extended to arbitrary $x$.

Let us discuss now the assumptions of Theorem 4. For positive (for $x \geqq x_{0}$ ) potentials we have not yet studied the continuity of $\psi(x, s)$ as $s \rightarrow 0$ along the real axis. It appears that $\psi(x, s)$ is continuous in $s$ in the whole half-plane $\operatorname{Im} s \geqq 0$. However, the proof of this assertion is impeded by the existence for arbitrary small $s>0$ (or $s<0$ ) of such a point $x$ (a turning point), where $q(x)=s^{2}$. In a neighbourhood of a turning point the asymptotics of $\psi(x, s)$ is described in terms of Eiry functions. Below (see Sect. 5) we shall prove the continuity of $\psi(x, s)$ as $s \rightarrow 0$ in the whole upper halfplane (and even in a broader region, including some part of the lower half-plane) for potentials, which admit an analytic continuation to some sector of a complex plane. For such potentials it is possible to avoid a rather cumbersome study of a neighbourhood of a turning point.

For negative (for $x \geqq x_{0}$ ) potentials we have excluded some neighbourhood of the imaginary axis, where e.g. the function $\psi(0, s)$ has an infinite number of zeros, corresponding to negative eigenvalues of $H$. On the other hand, in this case the first formula (3.5) permits us to evaluate the asymptotics of the spectral function $\rho(k)$ (see (2.23)) as $k \rightarrow 0$. Note that the function $v(k)$ defined by (2.20) equals now identically 1 . Hence solutions $\psi_{0}(x), \overline{\psi_{0}(x)}$ are linearly independent and, in particular, $\psi_{0}(x) \neq 0$ for $x \geqq 0$.

Corollary. Let the condition (3.1) hold and $q(x)<0$ for $x \geqq x_{0}$. Then

$$
\rho(k) \sim 2 \pi^{-1}\left|\psi_{0}(0)\right|^{-2} k, \quad k \rightarrow 0, \quad \psi_{0}(0) \neq 0 .
$$

\section{Analytic Continuation of the Spectral Function}

In this section it is assumed that the potential $q(x)$ is analytic in the region $\Gamma_{x_{0}}\left(\omega_{0}\right)=\left\{x \in \mathbb{C}:-\omega_{0}<\arg x<\omega_{0}, \quad|x|>x_{0}\right\}, \quad$ where $\omega_{0} \in(0, \pi / 2)$. Set 
$\Gamma_{x_{0}}^{+}\left(\omega_{0}\right)=\left\{x \in \mathbb{C}: 0 \leqq \arg x<\omega_{0},|x|>x_{0}\right\}$. The dependence of $\Gamma_{x_{0}}\left(\omega_{0}\right)$ and $\Gamma_{x_{0}}^{+}\left(\omega_{0}\right)$ on $\omega_{0}$ is usually dropped out of notation. By $\omega$ we denote the argument of a complex number $x$. Let us define analytic continuation (AC) of a function $f$, originally defined on $(\varepsilon, \infty), \varepsilon \geqq 0$, into a region $Y^{\varepsilon}[0, \delta)$ as a function that is analytic in $Y^{\varepsilon}(0, \delta)$, is continuous up to $(\varepsilon, \infty)$ and coincides there with $f$. An $\mathrm{AC}$ of $f$ is denoted by the same letter. First we shall study an analyticity of solutions

$$
\psi_{1}(x, s)=v(s)^{-1} \psi(x, s), \psi_{2}(x, s)=v(s)^{-1} \psi(x,-s)
$$

of Eq. (1.1), which are defined for $s>0$.

Theorem 5. Let the potential $q(x)$ be analytic in $\Gamma_{x_{0}}\left(\omega_{0}\right)$. Let relations $q(x) \rightarrow 0$, $|x| \rightarrow \infty$, and

$$
\int_{x_{0}}^{\infty}\left(\left|q^{\prime \prime}\left(r e^{i \omega}\right)\right|+\left|q^{\prime}\left(r e^{i \omega}\right)\right|^{2}\right) d r<\infty
$$

be fulfilled uniformly in $\omega \in\left(-\omega_{0}, \omega_{0}\right)$. Then the functions $\psi_{1}(x, s)$ for arbitrary $x \geqq 0$ and $\psi_{1}^{\prime}(x, s)$ for $x>0$ admit an $\mathrm{AC}$ in a sector $Y\left(-\omega_{0}, 0\right]$, and functions $\psi_{2}(x, s), x \geqq 0$, $\psi_{2}^{\prime}(x, s), x>0$, in a sector $Y\left[0, \omega_{0}\right)$. Moreover, for $x \geqq 0$ and $|s| \rightarrow \infty$

$$
\left.\begin{array}{l}
\psi_{1}(x, s) \sim(-i s)^{-1 / 2} \exp [i s(x-a)], s \in Y\left(-\omega_{0}, 0\right], \\
\psi_{2}(x, s) \sim(i s)^{-1 / 2} \exp [-i s(x-a)], s \in Y\left[0, \omega_{0}\right) .
\end{array}\right\}
$$

Relations (4.3) may be differentiated with respect to $x$ if $x>0$.

Proof. Let us consider for definiteness the function $\psi_{1}(x, s)$ and correspondingly the sector $Y\left(-\omega_{0}, 0\right]$. First for arbitrary $\varepsilon>0$ and $s \in Y^{\varepsilon}\left[0, \omega_{0}\right)$ we shall construct an AC of $\psi(x, s)$ and $\psi^{\prime}(x, s)$ to complex $x \in \Gamma_{x_{1}}^{+}$where $x_{1}=x_{1}(\varepsilon)$. Then for fixed $x \in \Gamma_{x_{1}}^{+}$ functions $\psi(x, s), \psi^{\prime}(x, s)$ will be analytically continued to $Y^{\varepsilon}(-\omega, 0]$. Hence it is easily deduced that $\psi(x, s), x \geqq 0, \psi^{\prime}(x, s), x>0$, admit an AC to $Y\left(-\omega_{0}, 0\right]$.

Let $\varepsilon>0$ be given and $x_{1}=x_{1}(\varepsilon)$ be chosen so that

$$
|Q(x, s)| \geqq c(\varepsilon)>0, \quad x \in \Gamma_{x_{1}}, \quad|s|>\varepsilon .
$$

Functions $Q(x, s)^{\gamma}(\gamma=1 / 2, \gamma=-1 / 4)$ are obviously analytic in $x \in \Gamma_{x_{1}}$ for fixed $s \in Y^{\varepsilon}\left(-\omega_{0}, \omega_{0}\right)$ and in $s \in Y^{\varepsilon}\left(-\omega_{0}, \omega_{0}\right)$ for fixed $x \in \Gamma_{x_{1}}$. Therefore

$$
\xi(x, y ; s)=\int_{x}^{y} Q(z, s)^{1 / 2} d z
$$

is also analytic separately in $x, y \in \Gamma_{x_{1}}, s \in Y^{\varepsilon}\left(-\omega_{0}, \omega_{0}\right)$. The contour of integration in (4.5) should belong to $\Gamma_{x_{1}}$, e.g. one may integrate over a piece of a straight line. Note an estimate

$$
\begin{aligned}
|\exp (-2 \xi(x, y ; s))| & \leqq C, x, y \in \Gamma_{x_{1}}, \arg x=\arg y \\
& =\omega \in\left[0, \omega_{0}\right), s \in Y^{\varepsilon}\left[-\omega+\delta, \omega_{0}\right),
\end{aligned}
$$

where $C$ depends only on $\varepsilon$ and $\delta$ ( $\delta$ is an arbitrary positive number).

Our construction of $\mathrm{AC}$ of $\psi(x, s)$ relies on the expression (2.6) of $\psi(x, s)$ in terms of $u_{j}(x, s), j=1,2$. For $s \in Y^{\varepsilon}\left(0, \omega_{0}\right), x \geqq x_{1}$ functions $u_{j}(x, s)$ are represented by convergent series (2.8). Functions $u_{j}^{(n)}(x, s)$ are easily studied by induction (see (2.9)) 
Namely, properties of $\zeta(y, s)$ and $\xi(x, y ; s)$ ensure that for fixed $s \in Y^{\varepsilon}\left(0, \omega_{0}\right)$ functions $u_{j}^{(n)}(x, s)$ are analytic in $x \in \Gamma_{x_{1}}^{+}$and the contour of integration in (2.9) can be deformed to the ray $\left(x, e^{i \omega} \infty\right)$. The integral (2.9) over $\left(x, e^{i \omega} \infty\right)$ is analytic in $s \in Y^{\varepsilon}\left(-\omega+\delta, \omega_{0}\right)$ and by $(4.4),(4.6) u_{j}^{(n)}(x, s)$ obey

$$
\begin{gathered}
\left|u_{j}^{(u)}(x, s)\right| \leqq C^{n}(n !)^{-1}\left[\int_{|x|}^{\infty}\left(\left|q^{\prime \prime}\left(r e^{i \omega}\right)\right|+\left|q^{\prime}\left(r e^{i \omega}\right)\right|^{2}\right) d r\right]^{n}, \\
x \in \Gamma_{x_{1}}^{+}, \quad s \in Y^{\varepsilon}\left[-\omega+\delta, \omega_{0}\right) .
\end{gathered}
$$

Thus according to (4.2) for $s \in Y^{\varepsilon}\left(0, \omega_{0}\right)$ functions $u_{j}(x, s)$ are also analytic in $x \in \Gamma_{x_{1}}^{+}$, and for $x \in \Gamma_{x_{1}}^{+}$they have an $\mathrm{AC}$ to $Y^{\varepsilon}\left(-\omega, \omega_{0}\right)$.

Let us return to the formula (2.6). The function

$$
\xi(a, x ; s)=\xi\left(a, x_{1} ; s\right)+\xi\left(x_{1}, x ; s\right), s \in Y^{\varepsilon}\left[0, \omega_{0}\right),
$$

is analytic in $x \in \Gamma_{x_{1}}$ and $\xi\left(x_{1}, x ; s\right)$ for fixed $x \in \Gamma_{x_{1}}$ is analytic in $s \in Y^{\varepsilon}\left(-\omega_{0}, \omega_{0}\right)$. Thus all factors in the right hand side of (2.6) are analytic in $x \in \Gamma_{x_{1}}^{+}$for $s \in Y^{\varepsilon}\left(0, \omega_{0}\right)$. By continuity the representation (2.6) for $x \in \Gamma_{x_{1}}^{+}$, is extended to real $s>\varepsilon$. Moreover, for $x \in \Gamma_{x_{1}}^{+}$all factors, except $\exp \left(-\xi\left(a, x_{1} ; s\right)\right)$, are analytic in $s \in Y^{\varepsilon}\left(-\omega, \omega_{0}\right)$. According to (4.1) it remains to prove the analyticity in $Y^{\varepsilon}\left(-\omega_{0}, 0\right]$ of $v(s) \exp \left(\xi\left(a, x_{1} ; s\right)\right)$ or by $(2.20)$ of

$$
2 g(s)-\xi\left(a, x_{1} ; s\right)=\xi\left(a, x_{1} ;-s\right), \quad s>\varepsilon .
$$

This follows from analyticity of $\xi\left(a, x_{1} ; s\right)$ in $Y^{\varepsilon}\left(\pi-\omega_{0}, \pi\right]$. Since $C$ in (4.7) tends to zero as $|s| \rightarrow \infty$, the function $\psi_{1}(x, s)$ obeys (4.3). By $(2.6) \psi_{1}^{\prime}(x, s)$ has similar properties. Now with the help of the formula $\psi_{1}(x, s)=b_{1}(s) \varphi(x, s)+d_{1}(s) \theta(x, s)$ it is easy to carry over the analyticity in $s \in Y^{\varepsilon}(-\omega, 0]$ of $\psi_{1}(x, s), \psi_{1}^{\prime}(x, s), x \in \Gamma_{x_{1}}^{+}$, to all $x \geqq 0$ for $\psi_{1}(x, s)$ and to $x>0$ for $\psi_{1}^{\prime}(x, s)$. Since $\varepsilon>0$ and $\omega<\omega_{0}$ are arbitrary, we find that $\psi_{1}(x, s), x \geqq 0, \psi_{1}^{\prime}(x, s), x>0$, are analytic in $s \in Y\left(-\omega_{0}, 0\right]$.

By Theorem 5 the equality $\rho^{-1}(s)=\pi(2 s)^{-1} \psi_{1}(0, s) \psi(0,-s), s>0$, implies that $\rho^{-1}(s)$ admits an $\mathrm{AC}$ to $Y\left(-\omega_{0}, 0\right]$. Moreover, according to (2.16), (4.3)

$$
\rho(s) \sim 2 \pi^{-1} s^{2}, \quad|s| \rightarrow \infty,
$$

for $s \in Y\left(-\omega_{0}, 0\right]$ and, in particular, $\rho^{-1}(s)$ does not have zeros for sufficiently large $|s|$. Similarly, the equality $\rho^{-1}(s)=\pi(2 s)^{-1} \psi_{2}(0, s) \psi(0, s), s>0$, ensures the same properties of $\rho(s)$ in $Y\left[0, \omega_{0}\right)$.

Theorem 6. Under the assumptions of Theorem 5 the spectral function $\rho(s)$ has a meromorphic continuation to the sector $Y\left(-\omega_{0}, \omega_{0}\right)$. Moreover, $\rho(s)$ is regular for sufficiently large $|s|$ and obeys (4.8) for $s \in Y\left(-\omega_{0}, \omega_{0}\right)$.

\section{The Asymptotics of the Spectral Function in the Complex Plane}

For the study of $\rho(s)$ as $s \rightarrow 0$ in some sector of the complex plane we need additional assumptions on $q(x)$, combining conditions of Sects. 3 and 4. First we shall show that under such assumptions the quasiclassical solution $\psi(x, s)$ of Eq. (1.1) admits an AC in $s$ to some sectors of a lower half-plane (for sufficiently small $|s|$ ) and is continuous there as $s \rightarrow 0$. 
Theorem 7. Suppose that $q(x)$ is analytic in $\Gamma_{x_{0}}$. Let $q(x) \neq 0$ for $x \in \Gamma_{x_{0}}$ and let relations

$$
\begin{gathered}
q(x) \sim q_{0} x^{-\alpha}, \alpha \in(0,2), q_{0}=\bar{q}_{0} \neq 0,|x| \rightarrow \infty, \\
\int_{x_{0}}^{\infty}\left[\left|q^{\prime \prime}\left(r e^{i \omega}\right)\right| r^{3 \alpha / 2}+\left|q^{\prime}\left(r e^{i \omega}\right)\right|^{2} r^{5 \alpha / 2}\right] d r<\infty
\end{gathered}
$$

hold uniformly in $\arg x=\omega \in\left(-\omega_{0}, \omega_{0}\right)$. Then in case $q_{0}>0$ for each $\delta>0$ and some $\varepsilon=\varepsilon(\delta)$ functions $\psi(x, s), x \geqq 0, \psi^{\prime}(x, s), x>0$, are analytic in $s \in Y_{\varepsilon}\left(-2^{-1} \alpha \omega_{0}+\delta\right.$, $\pi+2^{-1} \alpha \omega_{0}-\delta$ ). The relation (3.4) holds in the sector $Y\left[-2^{-1} \alpha \omega_{0}+\delta, \pi+2^{-1} \alpha \omega_{0}\right.$ $-\delta]$. In case $q_{0}<0$ functions $\psi(x, s), x \geqq 0, \psi^{\prime}(x, s), x>0$, are analytic in $s \in Y_{\varepsilon}\left(-\omega_{0}\right.$ $+\delta, \pi / 2) \cup Y_{\varepsilon}\left(\pi / 2, \pi+\omega_{0}-\delta\right)$, where as before $\varepsilon=\varepsilon(\delta)$ and $\delta$ is arbitrary. The first relation (3.5) holds in the sector $Y\left[-\omega_{0}+\delta, \pi / 2-\delta\right]$, and the second in the sector $Y\left[\pi / 2+\delta, \pi+\omega_{0}-\delta\right]$. Relations (3.4),(3.5) may be differentiated with respect to $x$ if $x>0$.

Proof of this theorem unifies considerations of Theorems 4 and 5. The properties of functions $\psi(x, s), \psi^{\prime}(x, s)$ in variable $s$ are previously investigated for complex $x$. Here we use essentially the absence of zeros of $Q(x, s)$ for suitable choice of $\omega=\arg x$ and sufficiently large $|x|$ if $s$ belongs to sectors specified in the formulation of the theorem. This permits us to perform on the ray $\arg x=\omega$ the same bounds as in Theorem 4. It follows that $\psi(x, s), \psi^{\prime}(x, s)$ are continuous as $s \rightarrow 0$ for $\arg x=\omega$ and consequently for positive $x$.

For definiteness we shall study an $\mathrm{AC}$ of $\psi(x, s), \psi^{\prime}(x, s)$ over positive half-axis. Let $q_{0}>0$. Let some positive number $\delta$ be given. By Theorem 4 it suffices to study $\psi(x, s)$ in a sector $Y\left(-2^{-1} \alpha \omega_{0}+\delta, \delta_{0}\right)$, where $\delta_{0}, \delta_{0}>0$, is arbitrary. According to (5.1) we choose $x_{1}=x_{1}(\delta) \geqq x_{0}$, so that

$$
|\arg x+\alpha \omega|<\delta, x \in \Gamma_{x_{1}} .
$$

Then for $x \in \Gamma_{x_{1}}^{+}, s \in Y\left(-2^{-1} \alpha \omega+\delta, \delta_{0}\right)$ (3.6) holds, where $c=c(\delta)$ does not depend on $\omega \in\left[0, \omega_{0}\right)$. Actually, denoting $A=s^{2}|q(x)|^{-1}$ and taking into account (5.3) one reduces (3.6) to $\left|1-e^{i \alpha \omega} A\right| \geqq c$. The last estimate is obvious, since $e^{i \alpha \omega} A \in Y\left(2 \delta, 2 \delta_{0}\right.$ $+\alpha \omega)$ and 1 does not belong to this sector. In particular, (3.6) implies that $Q(x, s) \neq 0$ if $x \in \Gamma_{x_{1}}^{+}, s \in Y\left(-2^{-1} \alpha \omega+\delta, \delta_{0}\right)$. Thus by continuity we define the regular branch of $Q(x, s)^{\gamma}(\gamma=1 / 2, \gamma=-1 / 4)$ first for complex $x \in \Gamma_{x_{1}}^{+}, s \in Y\left(0, \delta_{0}\right)$ and then for $s \in$ $Y\left(-2^{-1} \alpha \omega+\delta, \delta_{0}\right)$. It follows that $\xi(x, y ; s)$ for $s \in Y\left(0, \delta_{0}\right)$ may be analytically continued in $x$ and $y$ (separately) to the region $\Gamma_{x_{1}}^{+}$. Moreover, for fixed $x, y \in \Gamma_{x_{1}}^{+}, \arg x=\arg y=\omega>0$ the function $\xi(x, y ; s)$ is analytic in $s \in Y\left(-2^{-1} \alpha \omega\right.$ $\left.+\delta, \delta_{0}\right)$ and is continuous in this sector as $s \rightarrow 0$. Next we shall prove that for $x \in \Gamma_{x_{1}}^{+}, s \in Y\left(-2^{-1} \alpha \omega+\delta, \delta_{0}\right)$

$$
\operatorname{Re} e^{i \omega} Q(x, s)^{1 / 2}>0 .
$$

Since (5.4) holds for $x \geqq 0, s \in Y\left(0, \delta_{0}\right)$, it suffices to check that $\operatorname{Re} e^{i \omega} Q(x, s)^{1 / 2} \neq 0$. Supposing to the contrary, we find that $e^{2 i \omega} s^{2}=e^{2 i \omega} q(x)+\gamma^{2}$ for some $x \in \Gamma_{x_{1}}^{+}, s \in$ $Y\left(-2^{-1} \alpha \omega+\delta, \delta_{0}\right)$ and $\gamma=\bar{\gamma}$. By (5.3) $e^{2 i \omega} q(x) \in Y((2-\alpha) \omega-\delta,(2-\alpha) \omega+\delta)$ and consequently $e^{2 i \omega} q(x)+\gamma^{2}$ belongs to $Y(0,(2-\alpha) \omega+\delta)$. However, $e^{2 i \omega} s^{2}$ lies in the sector $Y\left((2-\alpha) \omega+2 \delta, 2 \delta_{0}+2 \omega\right)$, which does not intersect $Y(0,(2-\alpha) \omega+\delta)$. Thus 
$\operatorname{Re} e^{i \omega} Q(x, s)^{1 / 2} \neq 0$ and (5.4) holds. This in its turn implies (2.14) for $x, y \in \Gamma_{x_{1}}^{+}, \arg x$ $=\arg y=\omega, s \in Y\left(-2^{-1} \alpha \omega+\delta, \delta_{0}\right)$.

Now we use the representation (2.6). The function $\xi(a, x ; s)$ for $s \in Y\left(0, \delta_{0}\right)$ is analytic in $x \in \Gamma_{x_{1}}^{+}, \xi(a, x ; s)=\xi\left(a, e^{i \omega} x_{1} ; s\right)+\xi\left(e^{i \omega} x_{1}, x ; s\right)$ and $\xi\left(e^{i \omega} x_{1}, x ; s\right)$ is analytic in $s \in Y\left(-2^{-1} \alpha \omega+\delta, \delta_{0}\right)$. Moreover, since $q(x) \neq 0$ for $x \in \Gamma_{x_{0}}$, the function $\xi\left(a, e^{i \omega} x_{1} ; s\right)$ is also analytic in $s$ for sufficiently small $|s|$. Thus $\xi(a, x ; s)$ is analytic in $s \in Y_{\varepsilon}\left(-2^{-1} \alpha \omega+\delta, \delta_{0}\right)$ and is continuous in this sector as $s \rightarrow 0$. It remains to study $u_{j}(x, s), j=1,2$. As was shown in Sect. 4 , for fixed $s \in Y\left(0, \delta_{0}\right)$ functions $u_{j}(x, s)$ defined by (2.8) are analytic in $x \in \Gamma_{x_{1}}^{+}$if $|x|$ is sufficiently large. Functions $u_{j}^{(n)}(x, s)$ in (2.8) obey recurrent relations (2.9), where integrals are taken over the half-line $\left(x, e^{i \omega} \infty\right)$. Note that by virtue of estimates (3.6), (2.14) and conditions (5.1), (5.2), the formula (2.8) can be extended to all $x \in \Gamma_{x_{1}}^{+}$(we emphasize that as distinct from Sect. $4 x_{1}$ does not depend here on $|s|)$. Moreover, for $x \in \Gamma_{x_{1}}^{+}$functions $u_{j}^{(n)}(x, s)$ are analytic in $s \in$ $Y\left(-2^{-1} \alpha \omega+\delta, \delta_{0}\right)$ and are continuous in this sector as $s \rightarrow 0$. The series (2.8) converges and defines the AC of $u_{j}(x, s)$ to $Y\left(-2^{-1} \alpha \omega+\delta, \delta_{0}\right)$ that is continuous as $s \rightarrow 0$. Thus by (2.6) functions $\psi(x, s)$ and $\psi^{\prime}(x, s)$ for $x \in \Gamma_{x_{1}}^{+}$have the same properties in the region $Y_{\varepsilon}\left(-2^{-1} \alpha \omega+\delta, \delta_{0}\right)$. Now (2.17) implies that $\psi(x, s)$ for $x \geqq 0$ and $\psi^{\prime}(x, s)$ for $x>0$ are also analytic in $s \in Y_{\varepsilon}\left(-2^{-1} \alpha \omega+\delta, \delta_{0}\right)$ and are continuous as $s \rightarrow 0$ in this sector.

In the case $q_{0}<0$ the number $x_{1}$ should be chosen so that $|\arg q(x)+\alpha \omega-\pi|$ $<\delta$. Then estimates (3.6), (2.14) hold for $s \in Y\left(-\omega+\delta, \delta_{0}\right)$. All other considerations go through without any modifications.

Results of Theorem 7 determine the asymptotics of the spectral function $\rho(s)$ defined by (2.23). In the case $q_{0}<0,(3.8)$ obviously remains true for $s \in Y\left[-\omega_{0}\right.$ $\left.+\delta, \omega_{0}-\delta\right], s \rightarrow 0$. In the case $q_{0}>0$, note that by $(5.1) \psi_{0} \in L_{2}(0, \infty)$. Hence $\psi_{0}(0) \neq 0$ if $H$ does not have zero eigenvalue and, in particular, if $q(x) \geqq 0$ for all $x$ $\geqq 0$. It follows that $[\psi(0, s) \psi(0,-s)]^{-1} \rightarrow\left|\psi_{0}(0)\right|^{-2}$ as $s \rightarrow 0, s \in Y\left[-2^{-1} \alpha \omega_{0}\right.$ $\left.+\delta, 2^{-1} \alpha \omega_{0}-\delta\right]$. Thus it suffices to study the function $g(s)$ defined by (2.20). For sufficiently small $\varepsilon>0$ and $s \in Y_{\varepsilon}\left[-2^{-1} \alpha \omega_{0}+\delta, 2^{-1} \alpha \omega_{0}-\delta\right]$ the equation $Q(x, s)$ $=0$ has in $\Gamma_{x_{0}}$ a single root $\hat{x}(s)$ and $\hat{x}(s) \sim q_{0}^{1 / \alpha} s^{-2 / \alpha}, s \rightarrow 0$. Consequently, the definition (2.20) of $g(s), s>0$, can be rewritten in a form

$$
g(s)=\int_{a}^{\hat{x}(s)} Q(z, s)^{1 / 2} d z
$$

Thus $g(s)$ admits an $\mathrm{AC}$ to $Y_{\varepsilon}\left[-2^{-1} \alpha \omega_{0}+\delta, 2^{-1} \alpha \omega_{0}-\delta\right]$ by the formula (5.5), where an integral is taken e.g. over a piece of a straightline. Condition (5.1) ensures that $g(s) \sim g_{0} s^{1-2 / \alpha}, s \rightarrow 0$, where

$$
g_{0}=\left|q_{0}\right|^{1 / \alpha} \int_{0}^{1}\left(x^{-\alpha}-1\right)^{1 / 2} d x
$$

Moreover, if $q(x)$ obeys

$$
q(x)=q_{0} x^{-\alpha}\left[1+O\left(x^{-\beta}\right)\right], \beta>1-\alpha / 2,|x| \rightarrow \infty, x \in \Gamma_{x_{0}}\left(\omega_{0}\right),
$$

then $g(s) \sim g_{0} s^{1-2 / \alpha}+g_{1}+o(1), s \rightarrow 0$, where

$$
g_{1}=\int_{a}^{\infty}\left(|q(x)|^{1 / 2}-\left|q_{0}\right|^{1 / 2} x^{-\alpha / 2}\right) d x-\left|q_{0}\right|^{1 / 2} 2(2-\alpha)^{-1} a^{1-\alpha / 2} .
$$


So we have proved

Theorem 8. Let the conditions of Theorem 7 be fulfilled. Assume further that in the case $q_{0}>0$ zero is not an eigenvalue of the operator $H$ and (5.7) holds. Then for arbitrary $\delta>0$ and $s \rightarrow 0$

$$
\begin{aligned}
& \rho(s) \sim 2 \pi^{-1} s\left|\psi_{0}(0)\right|^{-2}, s \in Y\left[-\omega_{0}+\delta, \omega_{0}-\delta\right], q_{0}<0, \\
& \rho(s) \sim 2 \pi^{-1} s \exp \left(-2 g_{0} s^{1-2 / \alpha}-2 g_{1}\right)\left|\psi_{0}(0)\right|^{-2}, s \in Y\left[-2^{-1} \alpha \omega_{0}+\delta,\right. \\
& \left.\quad 2^{-1} \alpha \omega_{0}-\delta\right], q_{0}>0 .
\end{aligned}
$$

In particular, if conditions of Theorem 7 are fulfilled for some $\omega_{0}>0, q_{0}>0$ and zero is not an eigenvalue of $H$, then

$$
\rho(k) \sim 2 \pi^{-1}\left|\psi_{0}(0)\right|^{-2} k \exp \left[-2 \int_{a}^{\infty}\left(q(x)-k^{2}\right)_{+}^{1 / 2} d x\right], \quad k \rightarrow 0 .
$$

We emphasize that by its structure and physical meaning the last formula is similar to the classical Weyl's formula for the function of distribution of negative eigenvalues of $H$ in the case $q_{0}<0$.

\section{The Local Asymptotics at Large Times of the Solutions of the Time-Dependent Schrödinger Equation}

In this section we shall study the behaviour of the kernel $U(x, y ; t)$ of the operator $\exp (-H t) E(0, \infty)$ as $t \rightarrow \infty$. More precisely, we shall find the asymptotics of $U(x, y ; t)$ for fixed $x, y$ and $t \rightarrow \infty$ in the closed right half-plane. In particular, as $t \rightarrow \infty$ along the imaginary axis this gives the asymptotics of the kernel of the evolution operator for time-dependent Schrödinger equation (1.6). The projection $E(0, \infty)$ cancels the trivial part of the evolution operator corresponding to the discrete spectrum of $H$. According to (2.22) we shall proceed from the formula

$$
U(x, y ; t)=\int_{0}^{\infty} \varphi_{0}(x, s) \varphi_{0}(y, s) \exp \left(-s^{2} t\right) \rho(s) d s .
$$

Since $\varphi_{0}(x, s)$ is an entire function of $s$, the asymptotics of $U(x, y ; t)$ as $t \rightarrow \infty$ is determined by the behaviour of $\rho(s)$ as $s \rightarrow 0$.

In the case of negative (for $x \geqq x_{0}$ ) potentials the asymptotics of (6.1) may be easily found with the help of an obvious relation

$$
\int_{0}^{\infty} s f(s) \exp \left(-s^{2} t\right) d s \sim f(0)(2 t)^{-1}, \quad t=e^{i \tau}|t| \rightarrow \infty, \quad \tau \in(-\pi / 2, \pi / 2) .
$$

The formula (6.2) holds if e.g. $f(s)$ is continuous and $f(s)=O\left(e^{c s}\right), s \rightarrow \infty$. Thus under the assumptions of Theorem 4 by (2.2), (3.8), (4.8)

$$
U(x, y ; t) \sim \varphi_{0}(x, 0) \varphi_{0}(y, 0)\left(\pi\left|\psi_{0}(0)\right|^{2} t\right)^{-1},
$$

as $t=e^{i \tau}|t| \rightarrow \infty, \tau \in(-\pi / 2, \pi / 2)$. To treat the case $t= \pm i|t|$ one should use additionally the analyticity of $\rho(s)$ in some sector $Y\left(-\delta_{0}, \delta_{0}\right)$, which is ensured by Theorem 7. Then taking into account (2.2), (4.8), (5.9) one deforms the contour of 
integration to the ray $s=e^{i \delta}|s|$, where $\delta \in\left(0, \delta_{0}\right)$ for $t=-i|t|$ and $\delta \in\left(-\delta_{0}, 0\right)$ for $t=i|t|$.To the integral over $\left(0, e^{i \delta} \infty\right)$ the relation (6.2) is again applied.

The case of positive (for $x \geqq x_{0}$ ) potentials, when $\rho(s) \rightarrow 0$ exponentially as $s \rightarrow 0$, is essentially more difficult. Assume first that $t \rightarrow+\infty$. Let us split (6.1) in two integrals-over $(0, \varepsilon)$ and $(\varepsilon, \infty)$, where $\varepsilon=\varepsilon(t) \rightarrow 0$ as $t \rightarrow \infty$. The asymptotics (2.2), (4.8) show that an integral over $(\varepsilon, \infty)$ is $O\left(e^{-\varepsilon^{2} t}\right)$. In integral over $(0, \varepsilon) \rho(s)$ can be replaced by its asymptotics $(5.10)$ and $\varphi_{0}(., s)$ by $\varphi_{0}(., 0)$. Then with an error of an order $O\left(e^{-\varepsilon^{2} t}\right)$ the integral over $(0, \varepsilon)$ is again extended to $(0, \infty)$. This gives the relation

$$
\begin{aligned}
& U(x, y ; t)=\varphi_{0}(x, 0) \varphi_{0}(y, 0) 2 \pi^{-1} e^{-2 g_{1}}\left|\psi_{0}(0)\right|^{-2} \\
& \quad \times \int_{0}^{\infty} s \exp \left(-2 g_{0} s^{1-2 / \alpha}-s^{2} t\right) d s(1+o(1))+O\left(e^{-\varepsilon^{2} t}\right) .
\end{aligned}
$$

After the substitution $s=t^{-\alpha /(\alpha+2)} \sigma$ the asymptotics of the integral in (6.4) is evaluated by the Laplace method, i.e.

$$
\begin{aligned}
& \int_{0}^{\infty} s \exp \left(-2 g_{0} s^{1-2 / \alpha}-s^{2} t\right) d s \\
& \sim(2 \pi)^{1 / 2} s_{0} \vartheta^{\prime \prime}\left(s_{0}\right)^{-1 / 2} t^{-(3 \alpha+2) /(2 \alpha+4)} \exp \left[-\vartheta\left(s_{0}\right) t^{(2-\alpha) /(2+\alpha)}\right],
\end{aligned}
$$

where

$$
\vartheta(s)=2 g_{0} s^{1-2 / \alpha}+s^{2}, \quad s_{0}=\left[g_{0}(2-\alpha) \alpha^{-1}\right]^{\alpha /(\alpha+2)}
$$

$\left(s_{0}\right.$ is the minimum of $\left.\vartheta(s)\right)$. Suppose now that $t^{(2-\alpha) /(2+\alpha)}=o\left(\varepsilon^{2} t\right)$. Then $O\left(e^{-\varepsilon^{2} t}\right)$ vanishes faster than (6.5) as $t \rightarrow \infty$ and hence it may be omitted. Thus formulae (6.4)-(6.6) determine the asymptotics of $U(x, y ; t)$ as $t \rightarrow+\infty$.

If $t=e^{i \tau}|t| \rightarrow \infty, \tau \neq 0, \tau \in[-\pi / 2, \pi / 2]$, one should deform previously the contour of integration in (6.1). Let $\omega_{0}>2(\alpha+2)^{-1} \tau$. Then for sufficiently small $c>0$ by Theorems 6 and 8 one can integrate in (6.1) first over $\left(0, e^{-i \alpha \tau /(\alpha+2)} c\right)$ and then over the half-line $\left(e^{-i \alpha \tau /(\alpha+2)} c, e^{-i \alpha \tau /(\alpha+2)} c+\infty\right)$, which is parallel to the real axis. According to (2.2), (4.8) the second integral is $O\left(e^{-c_{1} t}\right), c_{1}>0$. The integral over $\left(0, e^{-i \alpha \tau /(\alpha+2)} c\right)$ can be treated similarly to the case $t \rightarrow+\infty$. So we have proved

Theorem 9.1) Let the assumptions of Theorem 4 hold and $q(x)<0$ for $x \geqq x_{0}$. Then the kernel $U(x, y ; t)$ of the operator $\exp (-H t) E(0, \infty)$ for fixed $x, y$ obeys $(6.3)$ as $t=e^{i \tau}|t|$ $\rightarrow \infty, \tau \in(-\pi / 2, \pi / 2)$. If for some $\omega_{0}>0$ the conditions of Theorem 7 are fulfilled and $q_{0}<0$, then (6.3) remains true for $t= \pm i|t| \rightarrow \infty$.

2) Let the assumptions of Theorem 8 hold and $q_{0}>0$. Then

$$
\begin{aligned}
U(x, y ; t) \sim & \varphi_{0}(x, 0) \varphi_{0}(y, 0) 2^{3 / 2} \pi^{-1 / 2} s_{0} \vartheta^{\prime \prime}\left(s_{0}\right)^{-1 / 2} e^{-2 g_{1}} \\
& \times\left|\psi_{0}(0)\right|^{-2} t^{-(3 \alpha+2) /(2 \alpha+4)} \exp \left[-\vartheta\left(s_{0}\right) t^{(2-\alpha) /(2+\alpha)}\right]
\end{aligned}
$$

as $t=e^{i \tau}|t| \rightarrow \infty$, where $\tau \in[-\pi / 2, \pi / 2],|\tau|<\omega_{0}(1+\alpha / 2)$, $\vartheta$ and $s_{0}$ are defined by(6.6), (5.6), $g_{1}$ by (5.8).

Note that formulae (6.3), (6.7) are uniform in $x, y$ from any compact interval. This ensures the local asymptotics as $t \rightarrow \pm \infty$ of the solution $u(x, t)$ of $(1.6)$ if the initial state $u_{0}(x)$ has finite support and is orthogonal to bound states of $H$. 


\section{The Asymptotics of Scattering Data. Discussion of Results}

As is well known, if $q(x)$ obeys

$$
\int_{x_{0}}^{\infty}|q(x)| d x<\infty
$$

then (1.1) has for $\operatorname{Im} s \geqq 0, s \neq 0$, the Jost solution $f(x, s)$ with the asymptotics (1.2) as $x \rightarrow \infty$. The function $f(x, s)$ for each $x \geqq 0$ is analytic in $s, \operatorname{Im} s>0$, and is continuous up to the real axis with the exception of point $s=0$. If $q(x)$ is analytic in $\Gamma_{x_{0}}$ and $q\left(r e^{i \omega}\right) \in L_{1}\left(x_{0}, \infty\right)$ uniformly in $\omega \in\left(-\omega_{0}, \omega_{0}\right)$, then $f(x, s)$ for each $x \geqq 0$ has an AC to the sector $Y\left(-\omega_{0}, \pi+\omega_{0}\right)$. Since $M(s)=f(0, s) \rightarrow 1$ as $s \rightarrow \infty, \operatorname{Im} s \geqq 0$, it is usually supposed that $\eta(k)=\arg M(k) \rightarrow 0, k \rightarrow \infty$. By continuity this uniquely determines the phase shift $\eta(k)$.

Under the assumption (7.1) for the pair of operators $H_{0}=-d^{2} / d x^{2}, H=$ $-d^{2} / d x^{2}+q(x)$ with the boundary condition $u(0)=0$ in the space $L_{2}(0, \infty)$, wave operators exist, are complete and the scattering operator is unitary. The scattering matrix $S(k)$, the partial scattering cross section $\sigma(k)$ and the physical wave function $\Psi(x, k)$ (normalized eigenfunction of the continuous spectrum) are expressed in terms of $M(k)$ by the following formulae:

$$
\left.\begin{array}{rl}
S(k) & =\exp (-2 i \eta(k)), \quad \sigma(k)=4 \pi k^{-2} \sin ^{2} \eta(k), \\
\Psi(x, k) & =M(k)^{-1} \varphi_{0}(x, k) .
\end{array}\right\}
$$

The function $\Psi(x, k)$ describes the scattering of the beam of particles with the energy $k^{2}$. The density of particles in the beam is supposed as usual to be equal to their velocity $2 k$. The quantity

$$
p(x, k)=\int_{0}^{x}|\Psi(x, k)|^{2} d x
$$

is interpreted as the number of particles, localized in the interval $(0, x)$. For potentials not obeying (7.1), the modulus $A(k)$ of $M(k)$ can be defined by the relation (1.3). This defines also $p(x, k)$.

We recall now for a comparison with slowly decreasing potentials some wellknown facts about the low energy behaviour of scattering data in a short-range case when $x q(x) \in L_{1}\left(x_{0}, \infty\right)$. In the latter case $M(s)$ is continuous as $s \rightarrow 0, \operatorname{Im} s \geqq 0$, $M(0)=\overline{M(0)}$ and generically $M(0) \neq 0$. If $M(0) \neq 0$, then $S(k) \rightarrow 1$ and $p(x, k)$ has finite (not vanishing) limit as $k \rightarrow 0$. The kernel $U(x, y ; t)$ of the evolution operator $\exp (-H t) E(0, \infty)$ obeys

$$
U(x, y ; t) \sim 2^{-1} \pi^{-1 / 2}|M(0)|^{-2} \varphi_{0}(x, 0) \varphi_{0}(y, 0) t^{-3 / 2}, \quad t \rightarrow \infty, \quad \operatorname{Re} t \geqq 0 .
$$

Moreover, if $x^{2} q(x) \in L_{1}\left(x_{0}, \infty\right)$, then $\eta(k) \sim \eta_{0} k$ and hence $\sigma(k) \rightarrow 4 \pi \eta_{0}^{2}$ as $k \rightarrow 0$. The following formula (Levinson's theorem) connects the number $N$ of negative eigenvalues of $H$ with the phase shift at zero energy:

$$
-\eta(0)=\pi N, \quad M(0) \neq 0 \quad(\eta(\infty)=0) .
$$

If $M(0)=0$, i.e., a zero-energy resonance exists, then $M(s) \sim M_{0} s, s \rightarrow 0$, and $N$ should 
be replaced by $N+1 / 2$ in (7.3). It follows that $U(x, y ; t)$ vanishes as $t^{-1 / 2}, t \rightarrow \infty$, and $S(k) \rightarrow-1, \sigma(k) \sim 4 \pi k^{-2}$ as $k \rightarrow 0$.

Let us return to the slowly decreasing case. Assume that both conditions (2.3) and (7.1) hold. Then the quasiclassical solution $\psi(x, s)$ and the Jost solution $f(x, s)$ exist. Now we shall establish the connection between them. Since $\left|Q(x, s)^{1 / 2}+i s\right|$ $\leqq C(s)|q(x)|$ for $\operatorname{Im} s \geqq 0, s \neq 0$, then by $(7.1)$

$$
\int_{a}^{x} Q(y, s)^{1 / 2} d y=-i s(x-a)+\int_{a}^{\infty}\left[Q(y, s)^{1 / 2}+i s\right] d y+o(1), \quad x \rightarrow \infty .
$$

Substituting (7.4) into (2.5) and comparing (2.5) with (1.2) we find that

$$
f(x, s)=(-i s)^{1 / 2} \exp \left\{\int_{a}^{\infty}\left[Q(y, s)^{1 / 2}+i s\right] d y+i s a\right\} \psi(x, s) .
$$

According to (7.5) and the results of Sects. 3 and 5 the asymptotics of $f(x, s)$ as $s \rightarrow 0$ ( $x$ fixed $)$ is determined by the function

$$
G(s)=\int_{a}^{\infty}\left[Q(x, s)^{1 / 2}+i s\right] d x .
$$

Note that if $q(x)<0, x \geqq x_{0}$, then $G(s)$ defined for $s \in Y[0, \pi / 2] \cup Y[\pi / 2, \pi]$ appears to be analytic in $Y(-\pi / 2, \pi / 2) \cup Y(\pi / 2,3 \pi / 2)$. If $q(x)>0, x \geqq x_{0}$, then $G(s)$ is analytic in $Y(0, \pi)$. Assume that $q(x)$ obeys $(5.7)$ with $\alpha \in(1,2)$. Then

$$
\begin{aligned}
& G(s)=-i G_{0} s^{1-2 / \alpha}-i g_{1}+o(1), \quad s \rightarrow 0, \quad s \in Y[-\pi / 2, \pi / 2], \\
& G(s)=i G_{0}(-s)^{1-2 / \alpha}+i g_{1}+o(1), \quad s \rightarrow 0, \quad s \in Y[\pi / 2,3 \pi / 2]
\end{aligned}
$$

in the case $q_{0}<0$, and

$$
G(s)=G_{0}(-i s)^{1-2 / \alpha}+g_{1}+o(1), \quad s \rightarrow 0, \quad s \in Y[0, \pi] .
$$

in the case $q_{0}>0$, where

$$
G_{0}=\left|q_{0}\right|^{1 / \alpha} \int_{0}^{\infty}\left[\left(x^{-\alpha}+1\right)^{1 / 2}-1\right] d x=g_{0}(\sin \pi / \alpha)^{-1},
$$

and $g_{0}, g_{1}$ are defined by (5.6), (5.8). The branch of $z^{\gamma}$ as before is fixed here by the condition arg $z \in(-\pi, \pi)$. If $q(x)$ is analytic in $\Gamma_{x_{0}}\left(\omega_{0}\right)$ and satisfies (5.7) with $q_{0}>0$ there, then $G(s)$ has an AC to $Y_{\varepsilon}\left(-2^{-1} \alpha \omega_{0}+\delta, \pi+2^{-1} \alpha \omega_{0}-\delta\right)$, where $\varepsilon=\varepsilon(\delta)$ and $\delta, \delta>0$, is arbitrary. Moreover in this case the asymptotics (7.7) remains true in the whole sector $Y\left[-2^{-1} \alpha \omega_{0}+\delta, \pi+2^{-1} \alpha \omega_{0}-\delta\right]$. Combining this information on the function $G(s)$ with Theorems 4 and 7 and taking (7.5) into account, we receive the following

Theorem 10. 1) Let $q(x)$ obey (5.7) with $\alpha \in(1,2)$ and (3.1). Then for each fixed $x \geqq 0$, $\delta>0$ and $s \rightarrow 0$

$$
\begin{aligned}
& f(x, s) \sim(-i s)^{1 / 2} \exp \left\{G_{0}(-i s)^{1-2 / \alpha}+g_{1}\right] \psi_{0}(x), \quad q_{0}>0, \quad s \in Y(\delta, \pi-\delta], \\
& f(x, s) \sim(-i s)^{1 / 2} \exp \left\{-i G_{0} s^{1-2 / \alpha}-i g_{1}\right] \psi_{0}(x), \quad q_{0}<0, \quad s \in Y[0, \pi / 2-\delta], \\
& f(x, s) \sim(-i s)^{1 / 2} \exp \left\{i G_{0}(-s)^{1-2 / \alpha}+i g_{1}\right] \overline{\psi_{0}(x)}, \quad q_{0}<0, \quad s \in Y[\pi / 2+\delta, \pi] .
\end{aligned}
$$


2) Let $q(x)$ be analytic in $\Gamma_{x_{0}}$ and let $q(x)$ obey (5.7), (5.2) with $\alpha \in(1,2)$ uniformly in $\omega=\arg x \in\left(-\omega_{0}, \omega_{0}\right)$. Then in the case $q_{0}>0$ the formula (7.8) remains true in the sector $Y\left[-2^{-1} \alpha \omega_{0}+\delta, \pi+2^{-1} \alpha \omega_{0}-\delta\right]$. In the case $q_{0}<0$ the formula (7.9) holds in $Y\left[-\omega_{0}+\delta, \pi / 2-\delta\right]$ and $(7.10)$ in $Y\left[\pi / 2+\delta, \pi+\omega_{0}-\delta\right]$.

Note that by (7.8) if $q_{0}>0$ the function $f(x, s)$ grows exponentially as $s \rightarrow 0$ in the closed upper half-plane. If $q_{0}<0$ the function $f(x, s)$ vanishes exponentially as $s=e^{i \gamma}|s| \rightarrow 0$ with $\gamma \in(0, \pi / 2)$ or $\gamma \in(\pi / 2, \pi)$. If $s \rightarrow 0$ along the real axis the exponent in (7.9) or (7.10) becomes purely oscillatory and hence $|f(x, s)|$ tends to zero as $|s|^{1 / 2}$.

Now we pick out of Theorem 10 results on the behaviour of the Jost function $M(k)$ as $k \rightarrow 0$ (the low energy asymptotics). We assume that in conformity with (2.16) the phase $\chi(k)$ of $\psi(0, k)$ is normalized by $\chi(k)=-k a+\pi / 4+o(1), k \rightarrow \infty$. Since $G(k) \rightarrow 0$ as $k \rightarrow \infty(\operatorname{see}(7.6))$, the relation (7.5) implies that $\eta(k)=\operatorname{Im} G(k)+\chi(k)+k a$ $-\pi / 4$.

Corollary. 1) Let the conditions of the first part of Theorem 10 hold and $q_{0}<0$. Then as $k \rightarrow 0$

$$
A(k) \sim\left|\psi_{0}(0)\right| k^{1 / 2}, \eta(k) \sim-G_{0} k^{1-2 / \alpha}-\eta_{-}+o(1), \eta_{-}=g_{1}+\pi / 4-\chi(0) .
$$

2) Let for some $\omega_{0}>0$ the conditions of the second part of Theorem 10 hold, $q_{0}>0$ and zero is not an eigenvalue of the operator $H$. Then as $k \rightarrow 0$

$$
\left.\begin{array}{l}
A(k) \sim k^{1 / 2} \exp \left(g_{0} k^{1-2 / \alpha}+g_{1}\right)\left|\psi_{0}(0)\right|, \\
\eta(k)=-G_{0} \cos \pi / \alpha \cdot k^{1-2 / \alpha}+\eta_{+}+o(1), \eta_{+}=-\pi / 4+\chi(0) .
\end{array}\right\}
$$

Note that according to (1.3) the asymptotics (7.11), (7.12) for $A(k)$ agree with the formulae (3.8), (5.10) for $\rho(k)$ and are valid for all $\alpha \in(0,2)$. Relation (7.11) ensures that in the case $q_{0}<0$ necessarily $A(0)=0$. Thus for negative slowly decreasing potentials the operator $H$ always has a zero-energy resonance (whereas for shortrange potentials this is an exceptional case). However, $A(k)$ vanishes only as $k^{1 / 2}$, i.e. slower than for short-range potentials. Hence this stable zero-energy resonance is "weaker" than an unstable one in a short-range case. In particular, this is exhibited in a local decay of $U(x, y ; t)$ : for negative slowly decreasing potentials $U(x, y ; t)$ vanishes as $t^{-1}$, whereas in a short-range case, as $t^{-3 / 2}(A(0) \neq 0)$ or $t^{-1 / 2}(A(0)=0)$. On the contrary, if $q_{0}>0$, relations (7.12) for $A(k)$ or (5.10) for $\rho(k)$ show that the density of quantum states vanishes exponentially as the energy tends to zero. This result may be interpreted as a disappearance of a sharp boundary at the spectral point zero between the continuous spectrum and the regular points of the operator $H$. In this case we call the point zero quasiregular. This notion is opposite to the notion of a zero-energy resonance when $\rho(k) / \rho_{0}(k) \rightarrow \infty$ as $k \rightarrow 0\left(\rho_{0}(k)=2 \pi^{-1} k^{2}\right.$ is the spectral function of $H_{0}$ ).

As is well known, the infinitude of the negative spectrum of the operator $H$ with the negative slowly decreasing potential is connected with the infinitude of zeros (see (3.2)) of the solution $\varphi_{0}(x, 0)$ of Eq. (1.1) for $s=0$. The above-described results and notions can be also clarified from the point of view of asymptotic behaviour of its solutions. In a short-range case $\varphi_{0}(x, 0)$ grows generically as $x$ and is bounded if a zero-energy resonance exists. For slowly decreasing negative potentials both solutions $\psi_{0}(x)$ and $\overline{\psi_{0}(x)}$ of $(1.1)$ for $s=0$ increase slower than $x^{\alpha / 4}=o\left(x^{1 / 2}\right), x \rightarrow \infty$, 
but are not bounded at infinity. The estimate $\varphi_{0}(x, 0)=o\left(x^{1 / 2}\right)$ corresponds to the existence of a zero-energy resonance. Since, however, $\varphi_{0}(x, 0)$ is not bounded at infinity, this resonance should be weaker than in a short-range case. For slowly decreasing positive potentials Eq. (1.1) for $s=0$ has solutions behaving itself as $\exp \left[ \pm q_{0}^{1 / 2} 2(2-\alpha)^{-1} x^{1-\alpha / 2}\right], x \rightarrow \infty$. Hence one of them is exponentially growing and another is exponentially decaying at infinity. This is similar to the behaviour of solutions of (1.1) for $\operatorname{Im} s>0$ and justifies the term "quasiregular point" for $s=0$.

Relations (7.11), (7.12) give also the asymptotics of the function $p(x, k)$ as $k \rightarrow 0$ :

$$
\begin{aligned}
& p(x, k) \sim k^{-1}\left|\psi_{0}(0)\right|^{-2} \int_{0}^{x}\left|\varphi_{0}(y, 0)\right|^{2} d y, \quad q_{0}<0, \\
& p(x, k) \sim k^{-1} \exp \left(-2 g_{0} k^{1-2 / \alpha}-2 g_{1}\right)\left|\psi_{0}(0)\right|^{-2} \int_{0}^{x}\left|\varphi_{0}(y, 0)\right|^{2} d y, \quad q_{0}>0 .
\end{aligned}
$$

These formulae are valid for all $\alpha \in(0,2)$. Thus for positive potentials decreasing at infinity slower than $x^{-2}$, the number of particles from the scattered beam, which are localized in a fixed compact region, decays exponentially as the energy of particles tends to zero. For Coulomb potential this circumstance was noted in [10].

The substitution of asymptotics (7.11), (7.12) for $\eta(k)$ in definitions (7.2) shows that the scattering matrix is oscillatory as $k \rightarrow 0$ and the partial scattering cross section obeys

$$
\sigma(k)=4 \pi k^{-2} \sin ^{2}\left(g_{ \pm} k^{1-2 / \alpha}+\eta_{ \pm}+o(1)\right),
$$

where signs "+" ("- ") correspond to positive (negative) potentials, $g_{+}=$ $-G_{0} \cos \pi / \alpha, g_{-}=G_{0}$.

Let us discuss the behaviour of $\eta(k)$ as $k \rightarrow 0$ from the point of view of its conformity with Levinson's theorem (formula (7.3)). In the case $q_{0}<0$, by (7.11) $\eta(k)$ tends to $-\infty$ as $k \rightarrow 0$, and the negative spectrum of $H$ is infinite. Thus for negative slowly decreasing potentials both sides of (7.3) equal $+\infty$. In the case $q_{0}>0,(7.12)$ shows that $\eta(k) \rightarrow+\infty$ as $k \rightarrow 0$, and hence for positive slowly decreasing potentials the formula (7.3) is violated.

In conclusion we note that all considerations of the present paper may be generalized from (1.1) to the equation $-u^{\prime \prime}+\ell(\ell+1) x^{-2} u+q(x) u=s^{2} u$. Since the centrifugal term $\ell(\ell+1) x^{-2}$ vanishes at infinity faster than $q(x)$, for arbitrary angular momentum $\ell$, the low energy asymptotics of all spectral data are similar to the case $\ell=0$. In particular, in contrast to short-range potentials for different $\ell$ partial scattering cross sections have the same order.

\section{References}

1. Landau, L. D., Lifshitz, E. M.: Quantum mechanics, Moscow: Fiz. Mat. 1963 (in Russian)

2. Newton, R. G. : Scattering theory of waves and particles. New York: McGraw-Hill 1966

3. Jensen, A., Kato, T.: Duke Math. J. 46, 583-611 (1979)

4. Jensen, A.: Manuscripta Math. 25, 61-77 (1978)

5. Rauch, J.: Commun. Math. Phys. 61, 149-168 (1978)

6. Yafaev, D. R.; Dokl. Akad. Nauk SSSR 258, 850-853 (1981) (Russian)

7. Yafaev, D. R.: Notes Sci. Sem. Steklov Math. Inst., Leningrad Branch Acad. Sci. USSR 51, 203-216 (1975) (Russian) 
8. Olver, F. W. J.: Introduction to asymptotics and special functions. New York, London: Academic Press 1974

9. Matveev, V. B., Skriganov, M. M.: Teor. Mat. Fis. 10, 238-248 (1972)(Russian)

10. Gamow, G.: Z. Phys. 51, 204 (1928)

Communicated by J. Ginibre

Received January 5, 1982 